\title{
Modelling Free Flowing Vehicular Traffic Noise
}

\author{
R.T. Sooriyaarachchi and D.U.J. Sonnadara
}

\begin{abstract}
Traffic noise of 650 vehicles classified into 8 vehicle classes were measured in several locations within the Western Province of Sri Lanka in order to extract the necessary coefficients to develop a road traffic noise prediction model. The model was developed to predict the traffic noise generated from free-flowing vehicles in roadways. Traffic flow data used for constructing this model was limited to vehicle noise, vehicle class, vehicle speed and the distance from the traffic flow line. It is shown that the predictions can be made within $\pm 11 \mathrm{~dB}(\mathrm{~A})$ accuracy with respect to the actual experimental observations. Microsoft.Net ${ }^{\circledast}$ platform was used for the development of the traffic simulator based on the model parameters.
\end{abstract}

Keywords: Road noise, Environment, Highways

\section{Introduction}

The highly industrialized living style of modern societies had produced a dramatic impact on the environment. The urbanization and adaptation characteristics of humans have made it difficult to identify the long-term impact of noise pollution. Today, environment noise has become a worldwide problem especially in metropolitan areas. The major contributor to the environment noise is the road traffic. Although the traffic noise is not usually loud enough to cause hearing problems, continuous exposure to unacceptable noise levels can create depression, sleep disturbance, annoyance and adverse health effects [1].

In a developing country such as Sri Lanka, not much attention has been paid to health hazards due to environment noise. Although the standards for the environment noise is well established [2-5], with the increasing traffic volume and frequent traffic congestion on urban roads, attention has often been paid to improve the traffic congestion rather than to arrest the noise pollution. The National Environmental Act No. 47 of 1980 [6] has set the maximum permissible noise levels at the boundary of the land in which any source of noise is located. Environmental Impact Assessment (EIA) of Colombo-Katunayaka Expressway Project, Southern Transport Development Project and proposed Outer Circular Highway Project has produced valuable data for background noise assessments [7-9]. However, independent research studies related to traffic noise pollution in Sri Lanka is scarce in literature.
Studies on modelling road traffic noise are available in literature [10-14]. These studies discuss the methodology adopted in the development of traffic noise prediction models for highways. The main focus of this paper is to test a simple model which depends on the spot speeds and distance of the vehicles to predict the noise generated by free-flowing traffic on local roadways.

\section{Methodology}

In this work traffic noise levels were measured in several selected sites located within the Western Province. Four sites having different road conditions that are similar to most of the roadways of Sri Lanka were selected. The factors that affect the modelling of traffic noise can be categorized as traffic factors, metrological factors and measuring site factors. A brief description of these factors that are relevant to this work is given below.

The vehicular traffic was divided into eight main groups, namely, Bus, Car, Double cab, Jeep, Lorry, Motor cycle, Three-wheeler and Van. It was assumed that only these vehicle types contribute to the road traffic noise and that all vehicles can be categorized into one of these classes. The noise levels vary within the selected categories due to their variations within the classes and the condition of the vehicles. Mode of operation of vehicles also affects the

Mr. R.T. Sooriyarachchi, Dip(IT) (SLIIT), B.Sc. Phys. (Hons) (Colonibo), Department of Plysics, Uniuersity of Colombo.

Prof. D.U.J. Sonnadara, C.Phys., MIP(SL), MCS(SL), B.Sc. Phys, (Hons) (Colombo), M.Sc. (Pittsburgh), Ph.D. (Pittsburgh), Department of Physics, Unizersity of Colombo. 
noise levels. For example, an accelerating vehicle produces a different level of noise than a vehicle moving at steady speed.

Sound levels are affected by meteorological conditions such as wind, temperature and humidity. To minimize the effect from the wind, a special windscreen made out of porous polyurethane sponge was used over the microphone. Atmospheric attenuation of the noise reduces the noise level when travelling through the air. Ambient temperature, relative humidity and ambient pressure influence the noise levels too. In all measuring sites air temperature was between $25-40^{\circ} \mathrm{C}$ and humidity was approximately $90 \%$.

The measuring site may have different conditions that could affect the noise measurement. The condition of the road, the absorption of noise waves by the ground and the reflection of sound by obstacles such as buildings, walls, trees, etc. would affect the measurement. The residual noise generated by other sound sources in the measuring area influences the noise level to a great extent. Thus, sites which have minimum influence due to external objects were selected.

It was decided to use the uncontrolled traffic approach. Six measuring distances perpendicular to the traffic flow line were selected, namely, $2.5 \mathrm{~m}$ (L1), $5.0 \mathrm{~m}$ (L2), $7.5 \mathrm{~m}$ (L3), $10.0 \mathrm{~m}$ (L4), $12.5 \mathrm{~m}$ (L5) and $15.0 \mathrm{~m}$ (L6). Noise and speed (over a range of $20 \mathrm{~km} / \mathrm{h}$ to $80 \mathrm{~km} / \mathrm{h}$ ) measurements were taken from isolated freeflowing vehicles for each distance. A RION NL04 Integrating sound level meter which has a bandwidth of $20-8000 \mathrm{~Hz}$ was used for noise level measurements.

All measurements were taken by placing the sound level meter at a height of $1.25 \mathrm{~m}$. Noise levels were measured in Leq (equivalent noise level) in units of $\mathrm{dB}$ on an a weighted scale in the time weighting mode 'fast' impulsive. Two markers were drawn on the road $\pm 100 \mathrm{~m}$ on either side of the measuring location and the average noise level of vehicles was recorded when vehicles travelled in between the marked region. The time duration varied from $9 \mathrm{~s}$ to $36 \mathrm{~s}$ for vehicles travelling at speeds of $80 \mathrm{~km} / \mathrm{h}$ to $20 \mathrm{~km} / \mathrm{h}$ respectively. For speed measurements, a digital radar speed gun which uses Doppler frequency shift technology to determine the spot speed was used. The gun allows taking speed measurements with $1 \mathrm{~km} / \mathrm{h}$ accuracy.

Before taking measurements, the sound meter must be calibrated. The sound meter NL- 04 has a calibration mode for this purpose. An internal $1000 \mathrm{~Hz}$ oscillator creates a signal with $94 \mathrm{~dB}$ sound level. Using this signal and the preset, the sound meter was calibrated before measurements were taken in the field.

Under uncontrolled approach, since the traffic flow is undisturbed and data is collected under real conditions, a more realistic model development is possible than when using the controlled approach.

\section{Results}

A total of 650 noise measurements were collected from eight different vehicle classes, namely, Buses, Cars, Double-cabs, Jeeps, Lories, Motor-Cycles, three-Wheelers and Vans. Contribution of each vehicle type to the data sample varied from a minimum of $8 \%$ (50 Jeeps) to a maximum of $16 \%$ (102 Vans).

Fitting a general noise model to the noise measurement data requires knowing the equation of the function that describes the variation of noise levels with dependent variables and initial estimation of the model coefficients. This can be carried out by fitting models to each vehicle type for each dependent variable individually by keeping the remaining variables fixed. Preliminary work showed that the noise variation with the distance of the vehicle is logarithmic and the noise variation with the speed of the vehicle is approximately linear [15]. Since these are the only two dependent variables, individual models were fitted for all 8 vehicle classes and rough estimation of the model coefficients for each vehicle class was extracted.

For the general design of the model, the statistical package S-PLUS ${ }^{\oplus}$ was used. In linear regression models, a numeric response variable, $y$, is modelled as a linear combination of predictor variables, $x_{i}$, for $j=1,2 \ldots, p$. Thus, the regression model has the form:

$$
y_{i}=\beta_{0}+\sum_{j-1}^{p} \beta_{j} x_{i j}+\varepsilon_{i}
$$


for $i=1,2, \ldots, n$, where $n$ is the number of observations, $\beta j^{\prime}$ 's are the coefficients to be extracted and $\varepsilon_{i}$ is the error for the $i^{\text {th }}$ observation. To make inferences regarding the fitted parameters of the model, the following assumptions were made; (1) $\varepsilon_{\mathrm{i}}$ are independent and identically distributed, (2) $\varepsilon_{i}$ have mean zero and finite variance, and, (3) $\varepsilon_{i}$ are distributed according to the normal distribution.

Least-squares best estimates of the coefficients in the model, $\beta j: j=0,1, \ldots, p$ are determined by minimizing the residual sum of squares relative to the coefficients. For the regression, measured noise values of vehicle types were given as the dependent variables and distance and speed as independent variables. Thus, the general formula for the developed model for equivalent noise level at a certain receiving point is:

$N=a+b V-c \ln (D)$

where $a, b$ and $c$ are constants, $V$ is the speed and $D$ is the distance. Final coefficients for model fitted parameters for each type of vehicle is given in table 1 .

The paired t-test was used for testing the model for goodness-of-fit. First, t-test was carried out for each type of vehicles individually. Paired $t$ test yields $t$-statistic values less than $5 \%$ significant level for all types of vehicles. That means, the predicted noise levels fit well with the measured traffic noise data for individual vehicles. Next, $t$-test was carried out for all the 650 data pairs together and a t-statistic value less than $5 \%$ significant level was observed. This implies that the noise levels fit well with the measured traffic noise data.

A dimensional test was also applied to test the model results (see figure 1). This method tests the deviation of data between the predicted and

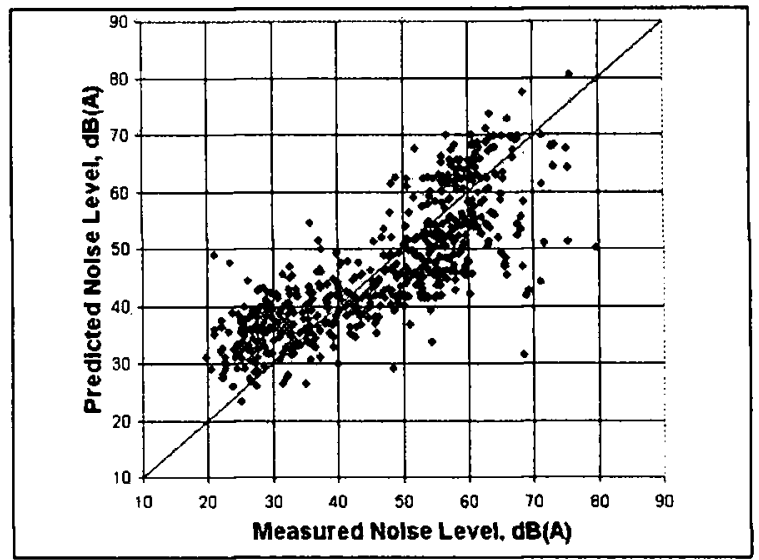

Figure 1: Correlation between the measured and predicted noise levels. A line is drawn at $45^{\circ}$ to show the ideal behaviour.

measured values of traffic noise from a 45 degree line. A slight deviation from the linear behaviour was observed. When the standard deviation of all 650 data pairs was taken, the model predictions were within $\pm 10.91 \mathrm{~dB}(\mathrm{~A})$ of the experimental observations.

The prediction accuracy obtained in the present work is not sufficient for practical use. The main contribution to the error within in any given vehicle class came from vehicle movement perpendicular to the traffic flow line. Since measurements were taken only from one side of the road, lateral movement of the vehicles across the road affected the noise measurements. In particular, the two measurement points $2.5 \mathrm{~m}$ and $5.0 \mathrm{~m}$ which are quite close to the traffic flow line can be affected easily due to this movement. This can be avoided by taking simultaneous measurements from both sides of the traffic route. Published work [10] has shown that with simultaneous measurements the prediction accuracy could be improved to \pm 3 $\mathrm{dB}(\mathrm{A})$ which could be the lower limit that can be achieved under controlled conditions. Another factor that affects the accuracy of the prediction

Table 1 - Fitted model parameters and their coefficients for each vehicle type.

\begin{tabular}{|l|c|c|}
\hline Vehicle Type & $\begin{array}{c}\text { Model Formula } \\
\text { (N=noise in dB(A), V=speed in } \mathbf{k m} / \mathbf{h}, \mathbf{D}=\text { distance in } \mathbf{m})\end{array}$ & \pm Std. Error dB(A) \\
\hline Bus & $N=53.07+0.777 \mathrm{~V}-16.58 \operatorname{Ln}(\mathrm{D})$ & 9.50 \\
Car & $N=44.56+0.505 \mathrm{~V}-12.84 \operatorname{Ln}(\mathrm{D})$ & 8.66 \\
Double-Cab & $N=60.05+0.401 \mathrm{~V}-17.32 \operatorname{Ln}(\mathrm{D})$ & 7.46 \\
Jeep & $N=67.23+0.221 \mathrm{~V}-13.24 \operatorname{Ln}(\mathrm{D})$ & 6.92 \\
Lorry & $N=79.89+0.181 \mathrm{~V}-20.06 \operatorname{Ln}(\mathrm{D})$ & 8.62 \\
Motorcycle & $N=56.42+0.448 \mathrm{~V}-15.48 \operatorname{Ln}(\mathrm{D})$ & 6.42 \\
Three-wheeler & $N=60.64+0.469 \mathrm{~V}-17.04 \operatorname{Ln}(\mathrm{D})$ & 8.27 \\
Van & $N=73.98+0.045 \mathrm{~V}-14.80 \operatorname{Ln}(\mathrm{D})$ & 8.47 \\
\hline
\end{tabular}


could be the measurement point at $2.5 \mathrm{~m}$ distance from the traffic flow line. At this distance the traffic flow cannot be considered as a linear source and the accuracy of the measurement becomes quite poor.

Using the developed model results, a software simulator was implemented to use as a tool to predict noise levels near traffic routes. The developed simulator software is capable of simulating noise due to volume of vehicles in eight vehicle classes with user specified mean speeds. The simulation calculates the real time combined noise levels at measuring points $L_{1^{\prime}}$ $\mathrm{L}_{2}, \mathrm{~L}_{3} \ldots \mathrm{L}_{6}$ and indicates the maximum noise levels observed for those points. The simulator is also capable of generating the noise contour maps in the vicinity of the traffic route and make predictions (see figure 2). Microsoft C\#.Net ${ }^{\circledast}$ platform was used as the development environment for the simulation software.

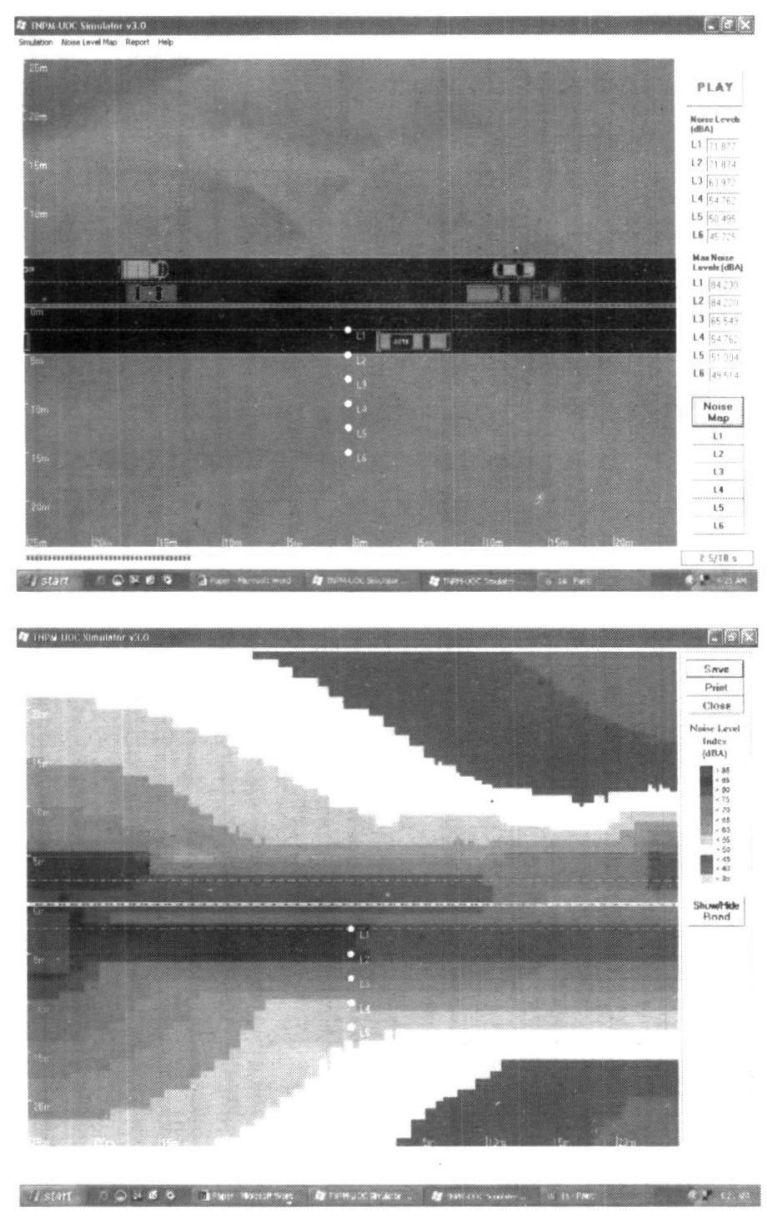

Figure 2: Screen captures of the software simulator for a given test scenario (a) Status of the traffic route (b) Noise contour map analysis.

\section{Conclusions}

In this work, the vehicular traffic noise behaviour with distance and spot speed was analyzed and presented for eight types of vehicles. The development of a simple model capable of predicting noise levels in the vicinity of vehicular routes for a given traffic volume was discussed. The work shows that the model is capable of predicting the combined traffic noise with varying traffic volumes, speeds and distances with accuracy better than $\pm 11 \mathrm{~dB}(\mathrm{~A})$. The major contributor to the noise is the variation within the vehicle classes and the lateral movement of the vehicles across the road perpendicular to the measurement. This is further influenced by the varying conditions of the vehicles and driving styles by the drivers.

Model was developed assuming an unobstructed free-flowing traffic movement. However, if the roadway has pedestrian crossing, traffic signal lights or even inclined sections, modifications to the model is required. Just by introducing a speed bump, the overall traffic noise could be increased by few decibels. This could be further complicated by the conditions and types of roads (normally, above $50 \mathrm{~km} / \mathrm{h}$ tire noise become dominant over the engine noise). With further improvements to reduce the prediction errors (such as applying ground attenuation rorrections, reflections due to buildings, taking simultaneous measurements, etc.) the model could be adapted to noise impact assessments near busy traffic routes.

\section{References}

1. World Health Organization, "Adverse health effects of noise", In Guidelines for community noise (Eds. Berglund, B., Lindvall, T., \& Schwela, D.), 1999, pp. 39-54, 1999.

2. International Organization for Standardization, "Acoustics - Description and measurement of environmental noise", Part 1: Basic quantities and procedures, ISO 1996-1: 1982[E], pp. 109-113.

3. International Organization for Standardization, "Acoustics - Description and measurement of environmental noise", Part 2: Acquisition of data pertinent to land use, ISO 1996-2: 1987[E], pp. 114120.

4. International Organization for Standardization, "Acoustics - Attenuation of sound during propagation outdoors", Part 1: Calculation of the absorption of sound by the atmosphere, ISO 96131: $1993[E]$, pp. $377-380$ 
5. International Organization for Standardization, "Acoustics - Measurement of noise emitted by passenger cars under conditions representative of urban driving", ISO 7188: 1994[E], pp. 125-129.

6. National Environmental Act No. 47 of 1980 , Government of Sri Lanka.

7. Industrial Technology Institute, "Background Noise Monitoring of Colombo-Katunayake Expressway Project", CP-43426, 2002.

8. Industrial Technology Institute, "Noise Monitoring of Southern Transport Development Project", CP-45557, 2003.

9. Industrial Technology Institute, "Monitoring of Background Noise Levels for Proposed Outer Circular Highway Project", CP-8186, 2005.

10. Suksaard, T., \& Sukasem, P., "Road Traffic Noise Prediction Model in Thailand", Applied Acoustics, Vol. 58, pp. 123-130, 1999.

11. Pamanikabud, P., \& Vivitjinda, P., "Noise prediction for highways in Thailand", Transportation Research D, Vol. 7, pp. 441-449, 2002.
12. Cho, D. S., Kim, J. H., Choi, T. M., Kim, B. H., \& Manvell, D., "Highway traffic noise prediction using method fully compliant with ISO 9613 : comparison with measurements", Applied Acoustics, Vol. 64, pp. 883-892, 2004.

13. El-Fadel, M., Shazbak, S., Baaj, M. H., \& Saliby, E., "Parametric sensitivity analysis of noise impact of multihighways in urban areas", Environmental Impact Assessment Review, Vol. 22, pp. 145-162, 2002.

14. Calixto, A., Diniz, F. B., \& Zannin, P. H. T, "Statistical modelling of road traffic noise in an urban setting", Cities, Vol. 20, No. 01, pp. 23-29, 2003.

15. Sooriyaarachchi, R. T., "Development of a road traffic noise prediction model", B.Sc. Thesis, Department of Physics, University of Colombo, 2005. 